\title{
Workspace Analysis of a Hybrid Kinematic Machine Tool with High Rotational Applications
}

\author{
Haiqiang Zhang, ${ }^{1}$ Hairong Fang $\mathbb{D}^{1,2}$ Yuefa Fang, ${ }^{1,2}$ and Bingshan Jiang ${ }^{1}$ \\ ${ }^{1}$ Robotics Research Center, School of Mechanical, Electronic and Control Engineering, Beijing Jiaotong University, Beijing, China \\ ${ }^{2}$ Key Laboratory of Vehicle Advanced Manufacturing, Measuring and Control Technology, Ministry of Education, Beijing, China \\ Correspondence should be addressed to Hairong Fang; hrfang@bjtu.edu.cn
}

Received 14 April 2018; Accepted 22 May 2018; Published 26 June 2018

Academic Editor: Carlos Llopis-Albert

Copyright (c) 2018 Haiqiang Zhang et al. This is an open access article distributed under the Creative Commons Attribution License, which permits unrestricted use, distribution, and reproduction in any medium, provided the original work is properly cited.

\begin{abstract}
This paper presents a novel parallel manipulator with one translational and two rotational (1T2R) degrees of freedom that can be employed to form a five-degree-of-freedom hybrid kinematic machine tool for large heterogeneous complex structural component machining in aerospace field. Compared with serial or parallel machine, hybrid machine has the merits of high stiffness, high speed, large workspace, and complicated surface processing ability. To increase stiffness, three-degree-of-freedom redundantly actuated and overconstrained 2PRU-PRPS parallel manipulator (P denotes the active prismatic joint) is proposed, which is utilized as the main body of hybrid machine. By resorting to the screw theory, the degree of freedom of the proposed mechanism is briefly addressed including the initial configuration and general configuration and validated by Grübler-Kutzbach (G-K) equation. Next, kinematic inverse solution and parasitic motion of the parallel manipulator are deduced and the transformational relations between the Euler angle and Tilt-Torsion (T-T) angle are identified. Thirdly, the performance evaluation index of orientation workspace is introduced, and the reachable workspace and joint workspace are formulated. Through specific examples, the reachable workspace, task workspace, and joint workspace of the redundant actuation parallel manipulator are depicted. Compared with overstrained 2PRU-PRS parallel manipulator, corresponding analyses illustrate that the proposed parallel manipulator owns much better orientation capability and is very meaningful to the development of the five-axis hybrid machine tool.
\end{abstract}

\section{Introduction}

Parallel kinematic manipulator tools were claimed to possess the inherent advantages such as high stiffness, high loading capability, high precision, low error accumulation, quick response speed, and high orientation capability. However, parallel manipulators suffer inherently from the unfavorable workspace. Therefore, it is of crucial significance to have a larger workspace so as to satisfy the working capability $[1,2]$. While the three-axis NC machine has more advantages in large workspace and good dexterity, however, it is only suitable for simple surface or small parts processing [3, 4]. The five-axis series CNC machine, adding two-degreeof-freedom rotating head attached to its mobile platform or two-degree-of-freedom rotary tables on the three-axis machine, can maintain the favourable orientation, but often scarified certain workspace, and may cause poor precision and stiffness by increasing the length of the actuator to enlarge the workspace $[5,6]$. So there is a contradiction between workspace and precision and stiffness for free surface machining by using the traditional series or parallel machine tool, and they cannot be able to satisfy requirements of the high speed milling for large heterogeneous complex surface in aerospace. At present, many complicated freedom surfaces are still milling manually, which inevitably has high cost, low efficiency, and long cycle, and the process not only depends heavily on the expertise and experience of the operators, but also requires much attention be given to processing; what is more, it is difficult to obtain high quality machining surface [7]. So it is of importance to explore a design approach for solving the required reachable workspace to envelope the task workspace, which will offer an ideal solution for machining [8].

Hybrid kinematic machine is expected to integrate the respective merits of pure serial and parallel machine, which has bigger workspace and better dynamic performance, 
higher precision and higher rigidity, more complicated surface processing ability, and more flexible orientation capability and has been successfully employed as machine tools and robots for high speed milling, drilling, and welding in aerospace and automotive industry for free surface processing, as well as assembly operations of aluminum structural parts [9]. It has been demonstrated practically by very successful applications such as a typical Sprint Z3 mechanism [10], Tricept hybrid machine tool [11], and Exechon hybrid machine tool [12]. In many practical applications, to increase the workspace of the three-degree-of-freedom parallel manipulator, we can add one or two long tracks. Simultaneously, to improve the orientation adjusting ability of the end effector, one can attach two- or three-degreeof-freedom rotating head and then form a multi-degree-offreedom hybrid machine tool with large workspace and high stiffness, as well as high orientation capability [13, 14].

Hybrid machine tool underwent fast improvements and drew particular interests for numerous researchers, since they satisfy the increasing demanding task requirements of many various applications such as in machine tools, assembly lines, and high speed machining used in automotive, railway, and construction industries. For instance, the German DS Technologie launched five-axis machining center Ecospeed spindle for aircraft structure components with complex geometries, and the spindle head was mounted on the end effector of parallel manipulator, which can realize rotation about the $\mathrm{x}$ - and $\mathrm{y}$-axis and translation along $\mathrm{z}$-axis, and translation in $\mathrm{X}$ and $\mathrm{Y}$ direction can be realized by two very long tracks [15]. Wang et al. [16] have proposed a 3SPR parallel mechanism which forms the main body of a 5DOF hybrid manipulator especially designed for high speed machining in the aircraft industry. A five-axis hybrid machine has been developed to realize lapping and milling for large complex structure component surface, and it is generated by serially adding a 2-DOF A/C-axis head to the coupling threedegree-of-freedom 3RPS parallel manipulator in terms of two rotations and one translation $[17,18]$. Hao et al. [19] came up with a novel two-degree-of-freedom parallel manipulator, incorporated a two-degree-of-freedom rotating head with $\mathrm{A}$ and $\mathrm{C}$ axis arrangement, and supplemented a mobile platform, which can form a gantry type five-axis hybrid machine tool to provide five-DOF movement capabilities. Huang et al. [20] put forward a practical 5-DOF hybrid reconfigurable manipulator module called Trivariant and built a variety of equipment to complete high speed milling for large aluminum structural components and complex molds. Wu et al. [21] have studied the three-degree-offreedom redundant actuation parallel manipulator, increased the freedom of movement and rotation in the worktable, and applied it to the five-degree-of-freedom hybrid machine tool to perform machining.

The remainder of the paper is organized as follows. The research background of hybrid parallel machine tools and traditional serial-parallel machines is presented firstly. In the subsequent section, the required degree of freedom for high speed milling machining of large heterogeneous complex freedom surface is briefly addressed, and a redundantly actuated and overconstrained 2PRU-PRPS parallel manipulator

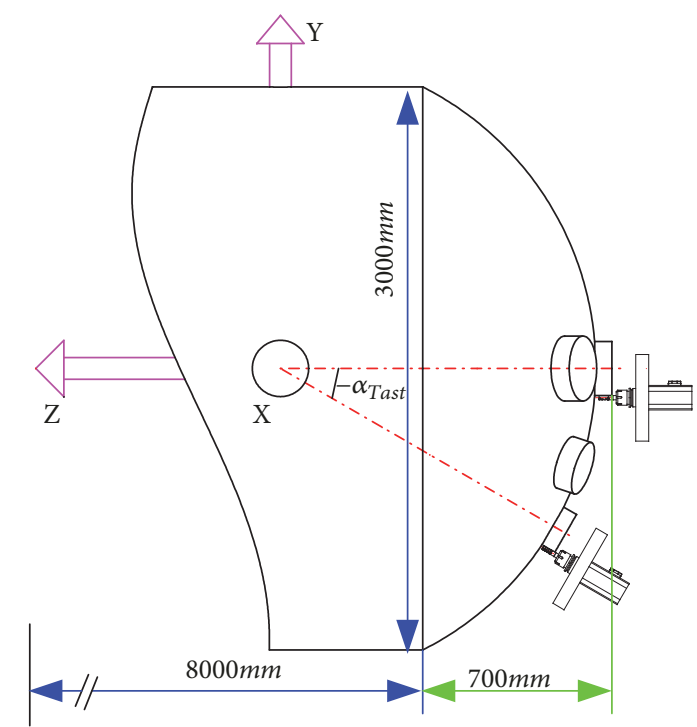

Figure 1: Limit rotational angle about $\mathrm{x}$-axis.

configuration is presented and selected as the main body of the hybrid machine tool. Afterward, the degree of freedom of the proposed parallel manipulator was analyzed including initial configuration and general configuration based on the screw theory, and the modified Grübler-Kutzbach (G$\mathrm{K})$ equation was utilized to verify the correctness for the degree of freedom. Then, the kinematic inverse position and transformational relation between Z-Y-X Euler angle and Tilt-Torsion (T-T) angle are performed. Next, based on the inverse position analysis and constraint conditions, the limit boundary searching algorithms and flowchart are introduced in detail. In the subsequent section, the parasitic motion of the parallel manipulator was carried out by numerical examples; simultaneously, the orientation workspace analysis of the parallel manipulator is performed and the reachable workspace, the task workspace, and the joint workspace of the parallel manipulator are intuitively depicted by using computer code programming. Finally, this article is concluded in "Conclusions" section.

\section{Design Requirements and Configuration of Hybrid Machine}

2.1. Function Requirement Analysis. The purpose of this paper is to design a hybrid machine tool used in the aerospace field for a large heterogeneous free surface high speed milling; the workpiece magnitude is shown in Figures 1 and 2, whose span in $\mathrm{x}$-axis is $3000 \mathrm{~mm}$ and in $\mathrm{y}$-axis is $3600 \mathrm{~mm}$, the total length of $\mathrm{z}$-axis is $9400 \mathrm{~mm}$, and the feed stroke of the end face in $\mathrm{Z}$ direction is $700 \mathrm{~mm}$, because the workpiece can only be placed flat and cannot be stood; therefore, the first condition of the overall layout selection of horizontal hybrid machine tool should be considered. In order to achieve the desired machining effects, the machine tool and surface normal are kept reasonable in the process of surface milling, so the machine tool should have at least five degrees of freedom, including three translational degrees and two 


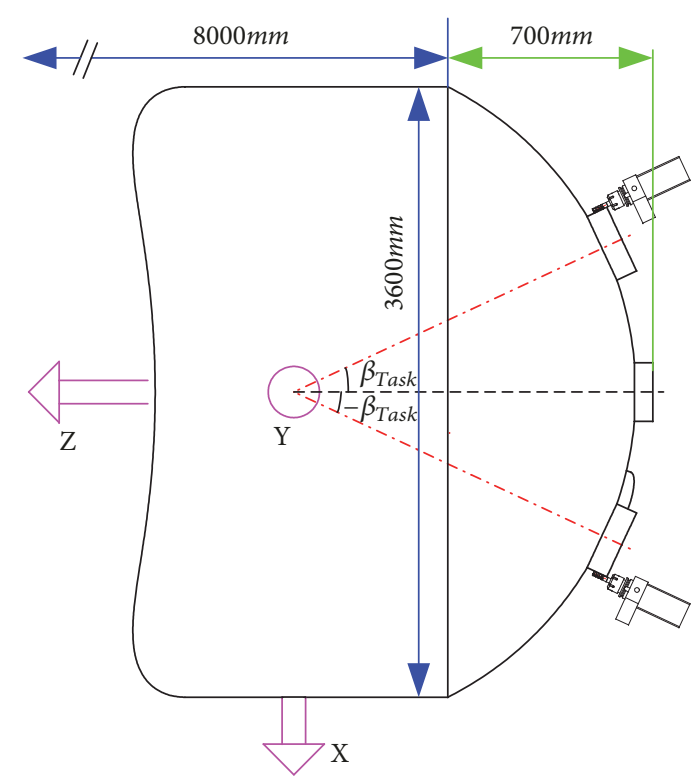

FiguRE 2: Limit rotational angle about y-axis.

rotational degrees. The latter is much more important than the former when it comes to perform three-dimensional milling [22]. Limit rotation angles (i.e., $\alpha_{\text {Task }}, \beta_{\text {Task }}$ ) between the tool and the surface during the curved surface machining are shown in Figures 1 and 2.

According to the characteristics of the curve surface, five relative motion between the surface and the cutter are processed, the $\mathrm{x}$ - and $\mathrm{y}$-axis motion are configured by the ball screw motion unit in series to satisfy the requirements of large workspace, and translation along $z$-axis and two rotations that are perpendicular to the $z$-axis are completed by parallel manipulator to meet the stiffness and orientation requirement of the tool. If the movements are achieved via traditional feed unit and orientation rotating head, then it cannot guarantee stiffness and accuracy of the machine owing to its cantilever. The specific design requirements are as follows:

(a) Absolute position accuracy: parallel manipulator configuration instead of traditional series configuration can reduce error accumulation.

(b) Normal precision: the parallel manipulator has good orientation capability to ensure the normal contact between the normal of free surface and machine tool point, so as to keep its normal position precision.

(c) Workspace: workspace is generated by adopting compound spherical joint to increase orientation workspace and serial X-Y long tracks to expand the position workspace of the hybrid machine tool.

(d) High stiffness: hybrid machine tool will produce heavy cutting force in the high speed machining process, so as to maintain higher machining accuracy, and the machine tool should be able to bear the heavy force and resist the external force deformation. Thus, the parallel manipulator should have higher stiffness characteristics. (e) High quality: the high orientation capability of parallel manipulator is employed instead of manual milling, which is beneficial to ensure the machining quality.

According to the above processing requirements, the parallel manipulator tool requires high stiffness and good orientation capability for the high speed machining of the free surface. Considering development trend of the hybrid machine tool, a five-axis hybrid machine tool can be constructed by adopting 1T2R three-degree-of-freedom parallel manipulator with two long $\mathrm{X}-\mathrm{Y}$ tracks, which is the best choice to realize the machining task requirements.

2.2. Configuration Design of the $1 T 2 R$ Mechanism. It is configuration innovation of the 1T2R three degrees of freedom that is the kernel of the hybrid machine tool. In order to complete the surface process with high efficiency and high precision, it is of crucial importance and significance for novel 1T2R lower-degree of freedom parallel manipulator with high stiffness, large workspace, and high orientation capability. There is an abundance of research on 1T2R mechanism. Kong and $\mathrm{Li}[23,24]$ divided the 1T2R parallel manipulator into three categories, one of which is PU configuration parallel manipulator, second kind of which is UP configuration parallel manipulator with coupling between rotation and movement, and the third is RPR configuration parallel manipulator, which can eliminate the coupling of rotation and movement and have certain space axis. Li et al. [25] pointed out that a class of one translation and two rotation DOFs parallel manipulator called [PP]S configuration mainly include 3PRS, 3-RPS, 3-RRS, and 3-PPS. Wang et al. [26] presented the 3-PUU parallel manipulator with rotational and translational coupling degrees of freedom; the difference between proposed mechanism and the 3PRS parallel manipulator is that the former did not have the spherical joint, but yet possesses much larger rotation angle and higher precision. Cui et al. [27] designed a 3RPS parallel manipulator with compound spherical joint that can increase the rotation angle. Li et al. [28] proposed a novel overconstrained parallel manipulator 2RPU\&SPR, the degree of freedom was analyzed based on the screw theory, and kinematic inverse position and Jacobian matrix were derived. Yan et al. [29] introduced a comparison study of the kinematics characteristics of two overconstrained 2-RPU\&SPR parallel manipulators. Xie et al. [30] conducted performance comparison analysis including motion force transmission performance, parasitic motion, and orientation capability of two overconstrained 2PRU-PRS and 2PRU-UPR parallel manipulator. Pashkevich [31] demonstrated that the overconstrained parallel manipulator can effectively improve the stiffness characteristic of the mechanism.

To increase the workspace of the parallel manipulator, enhance the kinematics performance of the machine tool, and improve the stiffness characteristic and dynamic characteristic, this paper adopts the redundantly actuated and overconstrained $2 \underline{P R U}-\underline{P R} \underline{P S}$ parallel manipulator with compound spherical joint as the main body of the hybrid machine tool. Simultaneously, to obtain a high rotation angle, using single limb double redundantly actuated technology, 


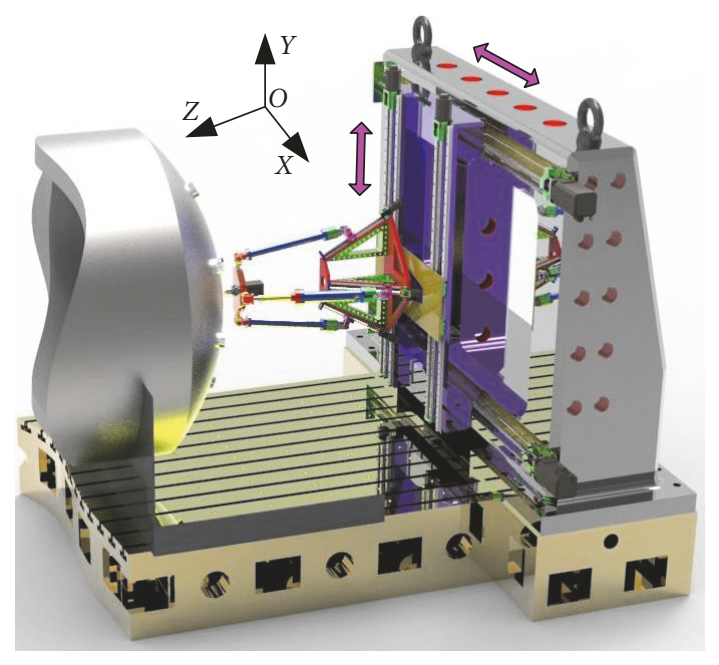

FIgure 3: A hybrid kinematic machine with five DOFs.

with two long X-Y tracks to form a 5-axis hybrid machine tool, it can be applied to machining for a large complex heterogeneous surface, and the overall structural concept is shown in Figure 3.

\section{Mobility Analysis of the 2PRU-PRPS Parallel Manipulator}

3.1. Architecture Description of the 2PRU-PRPS Parallel Manipulator. A novel redundantly actuated and overconstrained 2-PRU-PRPS parallel manipulator with compound spherical joint has been proposed in this paper, as shown in Figure 4, which is composed of a moving platform, a fixed base, and two identical constrained PRU chains and one double actuated PRPS chain together connecting the moving and fixed base. And two of PRU chains are symmetrically arranged and are located in a plane; furthermore two revolute axes are parallel with each other, and the second revolute axes of two Hooke joints are coincident and perpendicular to the third revolute axis of spherical joint. The parasitic motion appears only in an axial direction. The parallel manipulator is actuated by four active prismatic joints, and three actuators are fixed at the base, which reduces the inertia of the parallel manipulator tool. The spindle head is mounted at the end of the moving platform to complete the high speed milling.

To facilitate analysis, a fixed coordinate system $B-x y z$ is located at the center of the fixed base, and a moving coordinate system $A-u v w$ is attached at the center of the moving platform, respectively. Let the middle point of hypotenuse $B_{1} B_{2}$ be $B, x$-axis is perpendicular to $B_{1} B_{2}, y$-axis coincides with $B_{1} B_{2}$, and $z$-axis is perpendicular to the fixed base upward. Similarly, let the middle point of hypotenuse $A_{1} A_{2}$ be $A, u$-axis is perpendicular to $A_{1} A_{2}, v$-axis coincides with $A_{1} A_{2}$, and $w$ - axis is perpendicular to the moving platform upward. Without loss of generality, $\triangle B_{1} B_{2} B_{3}$ and $\triangle A_{1} A_{2} A_{3}$ are both isosceles right triangle, $\angle B_{1} B_{3} B_{2}=\angle A_{1} A_{3} A_{2}=90^{\circ}$, and their circumradii are nominated as $a$ and $b$, respectively. $B B_{1}=B B_{2}=B B_{3}=b$, and $A A_{1}=A A_{2}=A A_{3}=a$. With respect to $B$ $x y z$, the position vectors of points $B_{\mathrm{i}}$ and $A_{\mathrm{i}}(i=1,2,3)$ are as

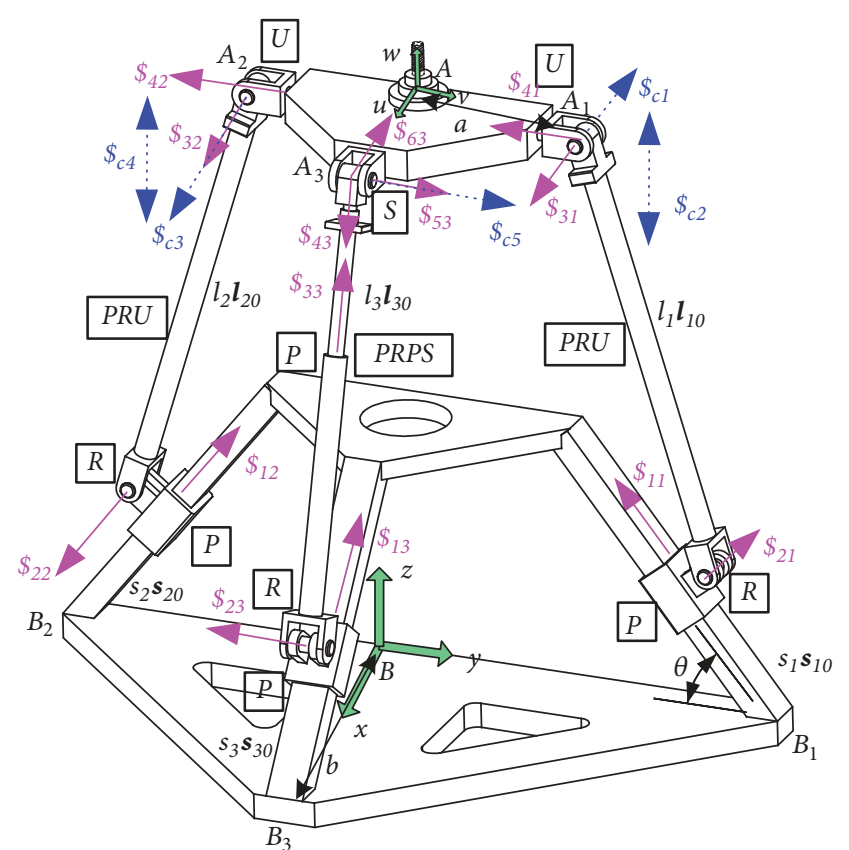

FIGURE 4: The schematic diagram of the 2-PRU-PRPS parallel manipulator.

follows. The coordinates of $B_{1}, B_{2}$, and $B_{3}$ are $\left(\begin{array}{lll}0 & b & 0\end{array}\right),\left(\begin{array}{lll}0 & -b\end{array}\right.$ 0 ), and ( $\left.\begin{array}{lll}b & 0 & 0\end{array}\right)$, respectively. The coordinates of $A_{1}, A_{2}$, and $A_{3}$ are $\left(\begin{array}{lll}x_{1} & y_{1} & z_{1}\end{array}\right),\left(\begin{array}{lll}x_{2} & y_{2} & z_{2}\end{array}\right)$, and $\left(\begin{array}{lll}x_{3} & y_{3} & z_{3}\end{array}\right)$, respectively. The layout angle of the fixed actuators is defined as $\theta$, and the angle between $B_{i}$ joint and $x$-axis of the coordinate system $B$-xyz is $\theta_{i}$.

3.2. Mobility Analysis of Initial Configuration. Lowermobility parallel manipulators whose independent degrees of freedom of the end effector are usually less than six can be implemented in many applications. In order to determine the motion pattern of the redundantly actuated and overconstrained 2PRU-PRPS parallel manipulator, mobility analysis is indispensable, whereas it is necessary to analyze the constraint screw provided by each chain to the moving platform and comprehensively analyze the constraint type of the moving platform.

It is assumed that the axes of the fixed coordinate system and the moving coordinate system are parallel to each other in the initial pose. Firstly, the first PRU limb is analyzed in the fixed coordinate system, and the twist screw of the chain can be expressed as

$$
\begin{aligned}
& \$_{11}=\left(\begin{array}{lllll}
0 & 0 & 0 ; 0 & -\mathrm{c} \theta & \mathrm{s} \theta
\end{array}\right) \\
& \$_{21}=\left(\begin{array}{lllll}
1 & 0 & 0 ; 0 & 0 & -b
\end{array}\right) \\
& \$_{31}=\left(\begin{array}{lllll}
1 & 0 & 0 ; 0 & z_{1} & -y_{1}
\end{array}\right) \\
& \$_{41}=\left(\begin{array}{lllll}
0 & -1 & 0 ; z_{1} & 0 & -x_{1}
\end{array}\right)
\end{aligned}
$$

where $s$ and $c$ are the abbreviation of sine and cosine, respectively. Employing reciprocal screw theory, the wrench system of (1) is obtained as 


$$
\begin{aligned}
& \$_{c 1}=\left(\begin{array}{lllll}
1 & 0 & 0 ; 0 & z_{1} & 0
\end{array}\right) \\
& \$_{c 2}=\left(\begin{array}{lllll}
0 & 0 & 0 ; 0 & 0 & 1
\end{array}\right)
\end{aligned}
$$

where $\$_{c 1}$ represents a constraint force passing $A_{1}$ point and parallel to $x$-axis and $\$_{c 2}$ represents a constraint couple perpendicular to the fixed base. by

Similarly, the twist screw of the second PRU limb is given

$$
\begin{aligned}
& \$_{12}=\left(\begin{array}{lllll}
0 & 0 & 0 ; 0 & c \theta & s
\end{array}\right) \\
& \$_{22}=\left(\begin{array}{lllll}
1 & 0 & 0 ; 0 & 0 & b
\end{array}\right) \\
& \$_{32}=\left(\begin{array}{lllll}
1 & 0 & 0 ; 0 & z_{2} & -y_{2}
\end{array}\right) \\
& \$_{24}=\left(\begin{array}{lllll}
0 & 1 & 0 ;-z_{2} & 0 & x_{2}
\end{array}\right)
\end{aligned}
$$

The wrench screw of (3) is obtained as

$$
\begin{aligned}
& \$_{c 3}=\left(\begin{array}{lllll}
1 & 0 & 0 ; 0 & z_{2} & 0
\end{array}\right) \\
& \$_{c 4}=\left(\begin{array}{lllll}
0 & 0 & 0 ; 0 & 0 & 1
\end{array}\right)
\end{aligned}
$$

where $\$_{c 3}$ represents a constraint force passing point $A_{2}$ and parallel to the $x$-axis of the fixed coordinate system and $\$_{c 4}$ represents a constraint couple perpendicular to the fixed base.

The twist screw of the third $\underline{P} \underline{P} S$ limb is given by

$$
\begin{aligned}
& \$_{13}=\left(\begin{array}{lllll}
0 & 0 & 0 ;-c \theta & 0 & s
\end{array}\right) \\
& \$_{23}=\left(\begin{array}{lllll}
0 & 1 & 0 ; 0 & 0 & b
\end{array}\right) \\
& \$_{33}=\left(\begin{array}{lllll}
0 & 0 & 0 ; l_{3} & 0 & n_{3}
\end{array}\right)
\end{aligned}
$$

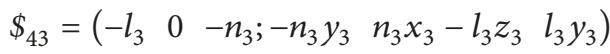

$$
\begin{aligned}
& \$_{53}=\left(\begin{array}{lllll}
0 & 1 & 0 ;-z_{3} & 0 & x_{3}
\end{array}\right) \\
& \$_{63}=\left(\begin{array}{lllll}
1 & 0 & 0 ; 0 & z_{3} & -y_{3}
\end{array}\right)
\end{aligned}
$$

where $l_{3}$ and $n_{3}$ are direction cosines of the three prismatic joints in limb 3 .

The wrench screw of (5) is obtained as

$$
\$_{c 5}=\left(\begin{array}{lllll}
0 & 1 & 0 ;-z_{3} & 0 & x_{3}
\end{array}\right)
$$

where $\$_{c 5}$ represents a constraint force passing point $A_{3}$ and parallel to $y$-axis of the fixed coordinate system.

The constraint screw of all the limbs acting on the moving platform can be obtained as

$$
\begin{aligned}
& \$_{c 1}=\left(\begin{array}{lllll}
1 & 0 & 0 ; 0 & z_{1} & 0
\end{array}\right) \\
& \$_{c 2}=\left(\begin{array}{lllll}
0 & 0 & 0 ; 0 & 0 & 1
\end{array}\right) \\
& \$_{c 3}=\left(\begin{array}{lllll}
1 & 0 & 0 ; 0 & z_{2} & 0
\end{array}\right) \\
& \$_{c 4}=\left(\begin{array}{lllll}
0 & 0 & 0 ; 0 & 0 & 1
\end{array}\right) \\
& \$_{c 5}=\left(\begin{array}{lllll}
0 & 1 & 0 ;-z_{3} & 0 & x_{3}
\end{array}\right)
\end{aligned}
$$

It is worth noting that $z_{1}=z_{2}=z_{3}=z$, and when the mechanism is initial configuration, the twist screw of the moving platform can be obtained by adapting the reciprocity of (7).

$$
\begin{aligned}
\$_{1}^{m} & =\left(\begin{array}{lllll}
0 & 1 & 0 ;-z & 0 & 0
\end{array}\right) \\
\$_{2}^{m} & =\left(\begin{array}{lllll}
1 & 0 & 0 ; 0 & z & 0
\end{array}\right) \\
\$_{3}^{m} & =\left(\begin{array}{lllll}
0 & 0 & 0 ; 0 & 0 & 1
\end{array}\right)
\end{aligned}
$$

where (8) represents the moving platform that is capable of rotation about $x$-and $y$-axes and translation along the $z$-axis.

3.3. Mobility Analysis of General Configuration. Similarly, the twist screw of the first PRU limb is given in the fixed coordinate system by

$$
\begin{aligned}
& \$_{11}=\left(\begin{array}{lllll}
0 & 0 & 0 ; 0 & -\mathrm{c} \theta & \mathrm{s} \theta
\end{array}\right) \\
& \$_{21}=\left(\begin{array}{lllll}
1 & 0 & 0 ; 0 & s_{1} s \theta & s_{1} c \theta-b
\end{array}\right) \\
& \$_{31}=\left(\begin{array}{lllll}
1 & 0 & 0 ; 0 & z_{1} & -y_{1}
\end{array}\right) \\
& \$_{41}=\left(\begin{array}{lllll}
0 & e_{1} & f_{1} ; f_{1} y_{1}-e_{1} z_{1} & -f_{1} x_{1} & e_{1} x_{1}
\end{array}\right)
\end{aligned}
$$

where $s_{i}$ denotes the twist of the $\mathrm{P}$ joint and $e_{i}$ and $f_{i}$ represent the direction cosine of the second revolute axis of the Hooke joint of $i$ limb.

Then, the wrench screw of limb 1 is easily obtained as

$$
\begin{aligned}
& \$_{c 1}=\left(\begin{array}{lllll}
e_{1} & 0 & 0 ; 0 & e_{1} z_{1}-f_{1} y_{1} & 0
\end{array}\right) \\
& \$_{c 2}=\left(\begin{array}{lllll}
0 & 0 & 0 ; 0 & -f_{1} & e_{1}
\end{array}\right)
\end{aligned}
$$

where $\$_{c 1}$ represents a constraint force passing point $A_{1}$ and parallel to $\$_{21}$; $\$_{c 2}$ represents a constraint couple perpendicular to $v$ and $\$_{31}$.

As previously mentioned, the twist screw of the second

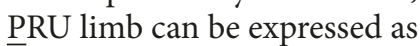

$$
\begin{aligned}
& \$_{12}=\left(\begin{array}{lllll}
0 & 0 & 0 ; 0 & c \theta & \mathrm{s} \theta
\end{array}\right) \\
& \$_{22}=\left(\begin{array}{lllll}
1 & 0 & 0 ; s_{2} s \theta & 0 & b-s_{2} c \theta
\end{array}\right) \\
& \$_{32}=\left(\begin{array}{lllll}
1 & 0 & 0 ; 0 & z_{2} & -y_{2}
\end{array}\right) \\
& \$_{24}=\left(\begin{array}{lllll}
0 & -e_{1} & -f_{1} ; e_{1} z_{2}-f_{1} y_{2} & f_{1} x_{2} & -e_{1} x_{2}
\end{array}\right)
\end{aligned}
$$

The wrench screw of (11) is obtained as

$$
\begin{aligned}
& \$_{c 3}=\left(\begin{array}{lllll}
e_{1} & 0 & 0 ; 0 & e_{1} z_{2}-f_{1} y_{2} & 0
\end{array}\right) \\
& \$_{c 4}=\left(\begin{array}{lllll}
0 & 0 & 0 ; 0 & -f_{1} & e_{1}
\end{array}\right)
\end{aligned}
$$

where $\$_{c 3}$ represents a constraint force passing point $A_{2}$ and parallel to $\$_{22} ; \$_{c 4}$ represents a constraint couple perpendicular to $v$ and $\$_{32}$. 
The twist screw of the third $\underline{P} \underline{P} S$ limb can be expressed as

$$
\begin{aligned}
& \$_{13}=\left(\begin{array}{lllll}
0 & 0 & 0 ;-c \theta & 0 & s \theta
\end{array}\right) \\
& \$_{23}=\left(\begin{array}{lllll}
0 & 1 & 0 ;-s_{3} s \theta & 0 & b-s_{3} c \theta
\end{array}\right) \\
& \$_{33}=\left(\begin{array}{lllll}
0 & 0 & 0 ; l_{3} & 0 & n_{3}
\end{array}\right)
\end{aligned}
$$

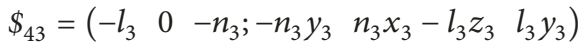

$$
\begin{aligned}
& \$ 53 \\
& =\left(\begin{array}{llll}
-n_{1} & e_{3} & l_{1} ; l_{1} y_{3}-e_{3} z_{3}-l_{1} x_{3}-n_{1} z_{3} & e_{3} x_{3}+n_{1} y_{3}
\end{array}\right)
\end{aligned}
$$

$\$ 63$

$$
=\left(\begin{array}{llll}
g_{1}-f_{1} & e_{1} ; e_{1} y_{3}+f_{1} z_{3} \quad g_{1} z_{3}-e_{1} x_{3}-f_{1} x_{3}-g_{1} y_{3}
\end{array}\right)
$$

where $g_{1}=\left(e_{1} l_{1}-f_{1} e_{3}\right) / n_{1}, e_{3}$ is a constant, and $l_{3}$ and $n_{3}$ represent the direction cosine of the third joint axis.

Subsequently, the wrench screw of (13) can be expressed as

$$
\$_{c 5}=\left(\begin{array}{lllll}
0 & 1 & 0 ;-z_{3} & 0 & x_{3}
\end{array}\right)
$$

where $\$_{c 5}$ represents a constraint force passing point $A_{3}$ and parallel to $\$_{23}$.

So far the constraint screw of the parallel manipulator can be expressed as

$$
\begin{aligned}
& \$_{c 1}=\left(\begin{array}{lllll}
e_{1} & 0 & 0 ; 0 & e_{1} z_{1}-f_{1} y_{1} & 0
\end{array}\right) \\
& \$_{c 2}=\left(\begin{array}{lllll}
0 & 0 & 0 ; 0 & -f_{1} & e_{1}
\end{array}\right) \\
& \$_{c 3}=\left(\begin{array}{lllll}
e_{1} & 0 & 0 ; 0 & e_{1} z_{2}-f_{1} y_{2} & 0
\end{array}\right) \\
& \$_{c 4}=\left(\begin{array}{lllll}
0 & 0 & 0 ; 0 & -f_{1} & e_{1}
\end{array}\right) \\
& \$_{c 5}=\left(\begin{array}{lllll}
0 & 1 & 0 ;-z_{3} & 0 & x_{3}
\end{array}\right)
\end{aligned}
$$

It is noteworthy that the direction vector $A_{1} A_{2}$ coincides with the axes of the second joint axis of the Hooke joint in the two PRU limbs, so the relation can be obtained as

$$
\frac{z_{2}-z_{1}}{y_{2}-y_{1}}=\frac{f_{1}}{e_{1}}
$$

By combining (16) and rearranging (15), the twist screw of the moving platform yields the following:

$$
\begin{aligned}
\$_{1}^{m} & =\left(\begin{array}{lllll}
1 & 0 & 0 ; 0 & z_{3} & 0
\end{array}\right) \\
\$_{2}^{m} & =\left(\begin{array}{lllll}
0 & e_{1} & f_{1} ; f_{1} y_{2}-e_{1} z_{2} & 0 & 0
\end{array}\right) \\
\$_{3}^{m} & =\left(\begin{array}{lllll}
0 & 0 & 0 ; 0 & 0 & 1
\end{array}\right)
\end{aligned}
$$

In (17), $\$_{1}^{m}$ represents one rotational degree of freedom passing point $A_{3}$ and parallel to $x$-axis, $\$_{2}^{m}$ represents one rotational degree of freedom of the moving platform passing point $A_{2}$ and parallel to the $v$-axis, and $\$_{3}^{m}$ represents a translational degree of freedom that is perpendicular to the fixed base. There are two instantaneous rotation axes, one of which is a straight line passing point $A_{3}$ and parallel to the $x$-axis and another is $v$-axis, located in the moving platform, and they change with the motion of the moving platform. It is worth noting that the mechanism has the same constraint screw and the degree of freedom with the 2PRU-PRS parallel manipulator.

Generally, the degree of freedom of parallel manipulator can be calculated by the modified Grübler-Kutzbach (G-K) equations; that is,

$$
F=d(n-g-1)+\sum_{i=1}^{g} f_{i}+v-\varsigma
$$

where $F$ represents the degree of freedom of the mechanism, $n$ represents the number of the components, $g$ represents the number of the kinematic joints, $d=6-\lambda$ represents the order of the mechanism, $f_{\mathrm{i}}$ represents the degree of freedom of the ith kinematic joint, $v$ represents the redundant constraints of the mechanism, and $\varsigma$ represents the local degree of freedom.

There was neither constraint couple in the same direction nor constraint force in collinearity among the constraint screw in the parallel manipulator; therefore, there is no common constraint, that is, $\lambda=0$. Because there are only three linearly independent variables in the five-constraint screw of the parallel manipulator, therefore, the parallel manipulator has two redundant constraints, that is, $v=$ 2. Because the parallel manipulator has no local degree of freedom, so $\varsigma=0$. We can see from the schematic of the mechanism that the number of the components is 6 , the number of the kinematic joints is 10 , and the relative freedom of all the kinematic joints in the mechanism is 14 . Due to the introduction of redundant actuation, there are five linear correlations between the six-twist screw, so there is a local degree of freedom, that is, $\varsigma=1[32,33]$.

Thus, based on the revised Grübler-Kutzbach (G-K) equations, the degree of freedom of the 2PRU-PRPS parallel manipulator can be recalculated as follows:

$$
\begin{aligned}
F & =d(n-g-1)+\sum_{i=1}^{g} f_{i}+v-\varsigma \\
& =6 \times(9-10-1)+14+2-1=3
\end{aligned}
$$

In summary, the redundantly actuated and overconstrained 2PRU-PRPS parallel manipulator has three degrees of freedom, i.e., two rotational degrees of freedom about $x$ axis and $v$-axis and one translational degree of freedom along $z$-axis.

\section{Inverse Kinematics of the Parallel Manipulator}

4.1. Position Inverse Analysis. The inverse kinematics solution is based on the determination of the structural parameters of the parallel manipulator, when the position and orientation of the moving platform are given, so as to solve the input displacement of the prismatic joints.

$Z$ - $Y$-X Euler angles are adopted to describe orientation matrix of the moving coordinate system with respect to the fixed coordinate system, first rotating the moving coordinate 
about $z$-axis by angle $\gamma$, then about $y$-axis of the new coordinate system by angle $\beta$, and finally about $x$-axis of the new coordinate system by angle $\alpha$. Thus, the orientation matrix $\mathbf{R}$ can be expressed as

$$
\begin{aligned}
\mathbf{R} & =\mathbf{R}(\gamma, z) \mathbf{R}(\beta, y) \mathbf{R}(\alpha, x) \\
& =\left[\begin{array}{ccc}
c \beta c \gamma & s \alpha s \beta c \gamma-c \alpha s \gamma & c \alpha s \beta c \gamma+s \alpha s \gamma \\
c \beta s \gamma & s \alpha s \beta s \gamma+c \alpha c \gamma & c \alpha s \beta s \gamma-s \alpha c \gamma \\
-s \beta & s \alpha c \beta & c \alpha c \beta
\end{array}\right]
\end{aligned}
$$

$\mathbf{p}=\left[\begin{array}{lll}x & y & z\end{array}\right]^{T}$ represents the position vector of the original point $A$ in the fixed coordinate system $B-x y z \cdot \boldsymbol{a}_{i}$ and $\boldsymbol{b}_{i}$ represent the position vector in the fixed coordinate of joints $A_{i}$ and $B_{i}$, and the coordinate of each joint in the fixed coordinate system can be, respectively, expressed in matrix form as

$$
\begin{aligned}
& \mathbf{a}_{1}=R\left(\begin{array}{lll}
0 & a & 0
\end{array}\right)^{T} \\
& \mathbf{a}_{2}=R\left(\begin{array}{lll}
0 & -a & 0
\end{array}\right)^{T} \\
& \mathbf{a}_{3}=R\left(\begin{array}{lll}
a & 0 & 0
\end{array}\right)^{T}, \\
& \mathbf{b}_{1}=\left(\begin{array}{lll}
0 & b & 0
\end{array}\right)^{T} \\
& \mathbf{b}_{2}=\left(\begin{array}{lll}
0 & -b & 0
\end{array}\right)^{T} \\
& \mathbf{b}_{3}=\left(\begin{array}{lll}
b & 0 & 0
\end{array}\right)^{T}
\end{aligned}
$$

Because of the arrangement of revolute joint in $\underline{P R U}$ and PRPS limbs, the center of Hooke joints and spherical joint cannot move along the axis of the revolute joint, so the following constraint conditions can be structured as

$$
\begin{aligned}
& \left(\mathbf{p}+\mathbf{a}_{1}\right)^{T} \cdot\left(\begin{array}{lll}
1 & 0 & 0
\end{array}\right)=0 \\
& \left(\mathbf{p}+\mathbf{a}_{2}\right)^{T} \cdot\left(\begin{array}{lll}
1 & 0 & 0
\end{array}\right)=0 \\
& \left(\mathbf{p}+\mathbf{a}_{3}\right)^{T} \cdot\left(\begin{array}{lll}
0 & 1 & 0
\end{array}\right)=0
\end{aligned}
$$

Selecting parameters $\alpha, \beta, z$ as three independent parameters, parasitic motion can be arranged as

$$
\begin{aligned}
& x=0 \\
& y=-a c \beta s \gamma \\
& \gamma=\arctan \left(\frac{s \alpha s \beta}{c \alpha}\right)
\end{aligned}
$$

The close-loop vector method is used to establish the equation of vector $A_{i} B_{i}$ in the fixed coordinate system $B$-xyz

$$
\mathbf{L}_{i}=\mathbf{a}_{i}+\mathbf{p}-\mathbf{b}_{i}=l_{i} \mathbf{l}_{i 0}+s_{i} \mathbf{s}_{i 1}
$$

Equation (24) squares on both sides; we arrange and obtain

$$
s_{i}^{2}-2 \mathbf{L}_{i}^{T} \mathbf{s}_{i 1} s_{i}+\mathbf{L}_{i}^{T} \mathbf{L}_{i}-l_{i}^{2}=0
$$

Position inverse solution of the 2ㅁU-PRPS parallel manipulator about $s_{i}$ in (25) can be expressed as follows [34].

$$
s_{i}=\mathbf{L}_{i}^{T} \mathbf{s}_{i 1} \pm \sqrt{\left(\mathbf{L}_{i}^{T} \mathbf{s}_{i 1}\right)^{2}-\mathbf{L}_{i}^{T} \cdot \mathbf{L}_{i}+l_{i}^{2}}
$$

where $\mathbf{s}_{i 1}=\left[\begin{array}{llll}-c \theta_{i} c \theta & -s \theta_{i} c \theta & s \theta\end{array}\right]^{T}, l_{1}=l_{2}=l$, and $l_{3}$ is an extensible and compressible link.

4.2. Orientation Description of T-T Angle. To better describe the orientation capability of the parallel manipulator, Liu and Bonev [35] pointed out that the [PP]S mechanism is three degrees of freedom with zero-torsion angle and systemically studied the relationships between different Euler angles and Tilt-Torsion (T-T) angle; that is, the orientation of the moving platform can be easily described as two variables: the azimuth angle and tilt angle. Therefore, T-T angle is a new orientation description method, which is usually more concise and more efficient to reflect the orientation capability of a class of 3-[PP]S mechanism compared with the description of Euler angles method. When the torsion angle is zero, the orientation matrix of T-T angle can be expressed as

$$
\begin{array}{r}
{ }^{T-T} \mathbf{R}(\varphi, \phi, 0)=\mathbf{R}_{z}(\varphi) \mathbf{R}_{y}(\phi) \mathbf{R}_{z}(-\varphi) \mathbf{R}_{z}(0) \\
=\left[\begin{array}{ccc}
s^{2} \varphi+c^{2} \varphi c \phi & s \varphi c \phi(c \phi-1) & c \varphi s \phi \\
s \varphi c \phi(c \phi-1) & c^{2} \varphi+s^{2} \varphi c \phi & s \varphi s \phi \\
-c \varphi s \phi & -s \varphi c \phi & c \phi
\end{array}\right]
\end{array}
$$

Combining (20) and (27), the T-T angles with zero-torsion can be converted to the Z-Y-X Euler angles via the following equation:

$$
\begin{aligned}
& \beta=a \sin (c \varphi s \phi) \\
& \alpha=a \sin \left(\frac{-s \varphi s \phi}{c \beta}\right) \\
& \gamma=a \sin \left(\frac{s \varphi c \phi(c \phi-1)}{c \beta}\right)
\end{aligned}
$$

Figures 5, 6, and 7 show the relations between $(\alpha, \beta, \gamma)$ and $(\varphi, \phi)$, separately. The circle coordinate denotes azimuth $\varphi$, and radial coordinate denotes the tilt angle $\phi$.

\section{Orientation Workspace Analysis}

The orientation workspace of parallel manipulator tool is an important performance index, which is the set of all practically feasible orientation of the moving platform. By analyzing the workspace, we can deduce the problem whether the expected machining range can be realized. The main analysis methods contain analytical method and numerical method; in this paper, the limit boundary searching method of reachable workspace was adopted, and the mechanism is divided into several single limbs, and the boundary of single limb space is obtained by using the surface enveloping theory; finally, the whole workspace of the mechanism is obtained by using the surface intersection technique. The difficulty of the 
$-60$

$-30$

0

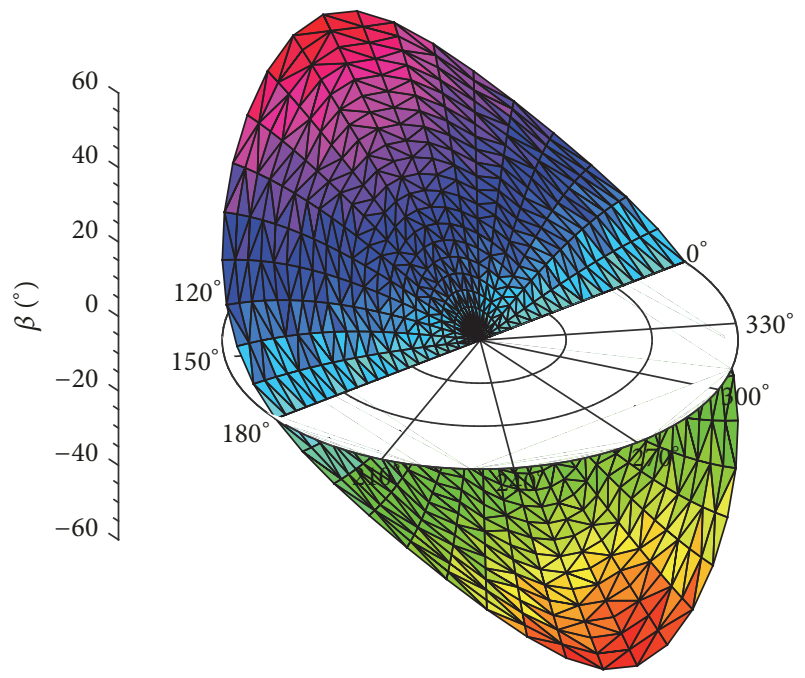

Figure 5: The relation of $\beta$ referring to $(\varphi, \phi)$.

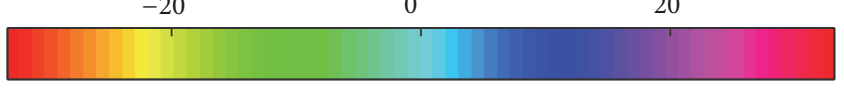

FIGURE 6: The relation of $\alpha$ referring to $(\varphi, \phi)$.

$-3$
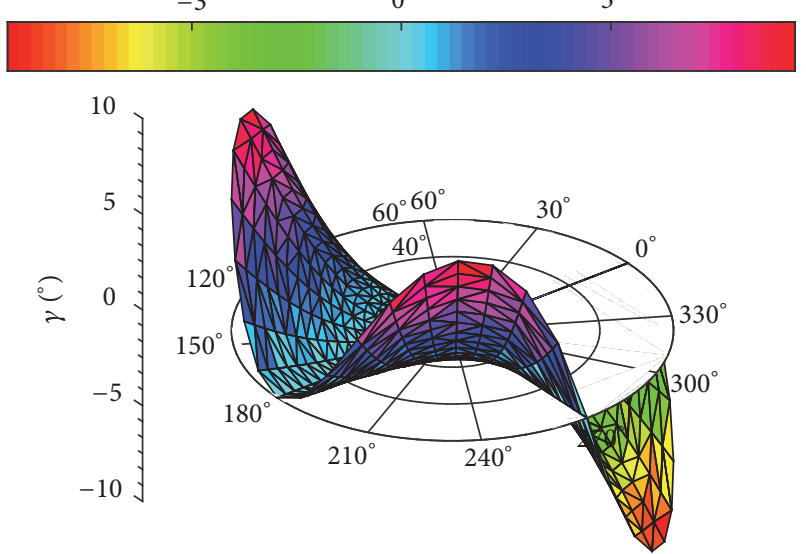

FiguRE 7: The relation of $\gamma$ referring to $(\varphi, \phi)$.
60 parallel manipulator, while the workspace analysis of parallel manipulator is mainly to investigate the reachable workspace of the end effector. For 1T2R parallel manipulator, the workspace of the manipulator mainly considers the rotational capability about the $x$-axis and the $v$-axis and the translational capability along $z$-axis. Over the past decade, workspace was investigated by many scholars with different methods and algorithms. Referring to [36], a discrete boundary searching method was implemented to calculate the workspace of parallel manipulator considering the driving constraint and joints constraint in the polar coordinate system. Pond [37] investigated reachable workspace and dexterous workspace of three parallel manipulators (including 3-PRS, 3-RPS, and Tricept) based on homogeneous Jacobian matrix condition number and conducted quantitative analysis on the workspace. Herrero et al. [38] solve the workspace of 2PRU-1PRS parallel manipulator based on the calculation of inverse kinematics and geometrical constraint and singularity constraint; what is more, they pointed out that maximum inscribed ball can be utilized to measure the workspace. Fu and Gao et al. [39] obtained the position workspace and orientation workspace of the parallel manipulator with decoupling three branches of six degrees of freedom by using a numerical searching method and chose the maximum inscribed cylinder and sphere envelope as the specified shade in the workspace.

5.1. Parameters Constraint Condition. In the actual machining process of parallel manipulator tool, the following limitations should be considered.

(1) Limitations of the Actuated Joints $s_{i}$

$$
s_{\min } \leq s_{i} \leq s_{\max }
$$

where $s_{\min }$ and $s_{\max }$ represent the minimum and maximum stroke of active joint, respectively. Here, $s_{\min }=0$ and $s_{\max }=$ $650 \mathrm{~mm}$.

(2) Limitation of Redundantly Actuated Joint $q_{4}$

$$
q_{\min } \leq q_{4} \leq q_{\max }
$$

where $q_{\min }$ and $q_{\max }$ represent the minimum and maximum displacement of the second-stage active joint of the third limb, respectively. Here $q_{\min }=900 \mathrm{~mm}$ and $q_{\max }=1200 \mathrm{~mm}$.

\section{(3) Joint Angle Constraints}

(a) The Rotation Angle $R_{2 i}$ of the Rotation Joint $R$ Should Be Satisfied

$$
R_{2 \min } \leq R_{2 i}=a \cos \left(\frac{s_{1 i} \cdot l_{i 0}}{\left|s_{1 i}\right|\left|l_{i 0}\right|}\right) \leq R_{2 \max }
$$


where $R_{2 \min }$ and $R_{2 \max }$ represent the minimum and maximum limit angles of the rotation joint $\mathrm{R}$ of the $i$ th limb, respectively. Here $R_{2 \min }=0$ and $R_{2 \max }=\pi / 6$.

(b) The Rotation Angle $R_{3 i}$ of the First Revolute Axis of the Hooke Joint

$$
R_{3 \min } \leq R_{3 i}=a \cos \left(\frac{s_{4 i} \cdot l_{i 0}}{\left|s_{4 i}\right|\left|l_{i 0}\right|}\right) \leq R_{3 \max }
$$

where $R_{3 \min }$ and $R_{3 \max }$ represent the minimum and maximum angle of the first revolute axis of the Hooke joint of the $i$ th limb, respectively. Here $R_{3 \min }=\pi / 6$ and $R_{3 \max }=\pi$.

(c) The Rotation Angle $R_{53}$ of the Second Revolute Axis of the Compound Spherical Joint Should Be Satisfied

$$
R_{4 \min } \leq R_{53}=a \cos \left(\frac{s_{6 i} \cdot l_{i 0}}{\left|s_{6 i}\right|\left|l_{i 0}\right|}\right) \leq R_{4 \max }
$$

where $R_{4 \min }$ and $R_{4 \max }$ represent the minimum and maximum angles of the second revolute axis of the compound spherical joint, respectively. Here $R_{4 \min }=\pi / 9$ and $R_{4 \max }=\pi$.

(4) Interference Constraints of the Kinematic Limb. Because the parallel manipulator once determines limitation of the revolute joint $\mathrm{R}$, there is no interference between adjacent links, so the interference constraint cannot be taken into account.

(5) Singularity Configuration Constraint. The singularity configuration of the parallel manipulator will seriously affect the kinematic performance of the parallel manipulator, and those configurations should be avoided. So the rank of the constraint screw system is bound to be three; that is,

$$
\operatorname{Rank}\left(\$_{c 1}, \$_{c 2}, \$_{c 3}, \$_{c 4}, \$_{c 5}\right)=3
$$

5.2. Workspace Algorithm. The orientation workspace of the manipulator tool is generated according to the structure parameters and constraint conditions of the parallel manipulator aforementioned. Based on the inverse position solution, the constraint conditions of each step are judged, respectively, and the detailed search algorithm is seen in the flowchart as shown as Figure 8. If the condition is satisfied, the feasible points are recorded; on the contrary, the others are discarded. The singularity-free reachable workspace and task workspace of the 2PRU-PRPS parallel manipulator have been generated by adopting Matlab programming. Simultaneously, a series of displacement of the active prismatic joint can be obtained, whose set is called joint workspace.

\section{Application Examples and Performance Analysis}

Numerical examples are presented to verify the validity of the theory, and its main structure parameters of the redundant actuation parallel manipulator are as follows (unit: $\mathrm{mm}$ ). The

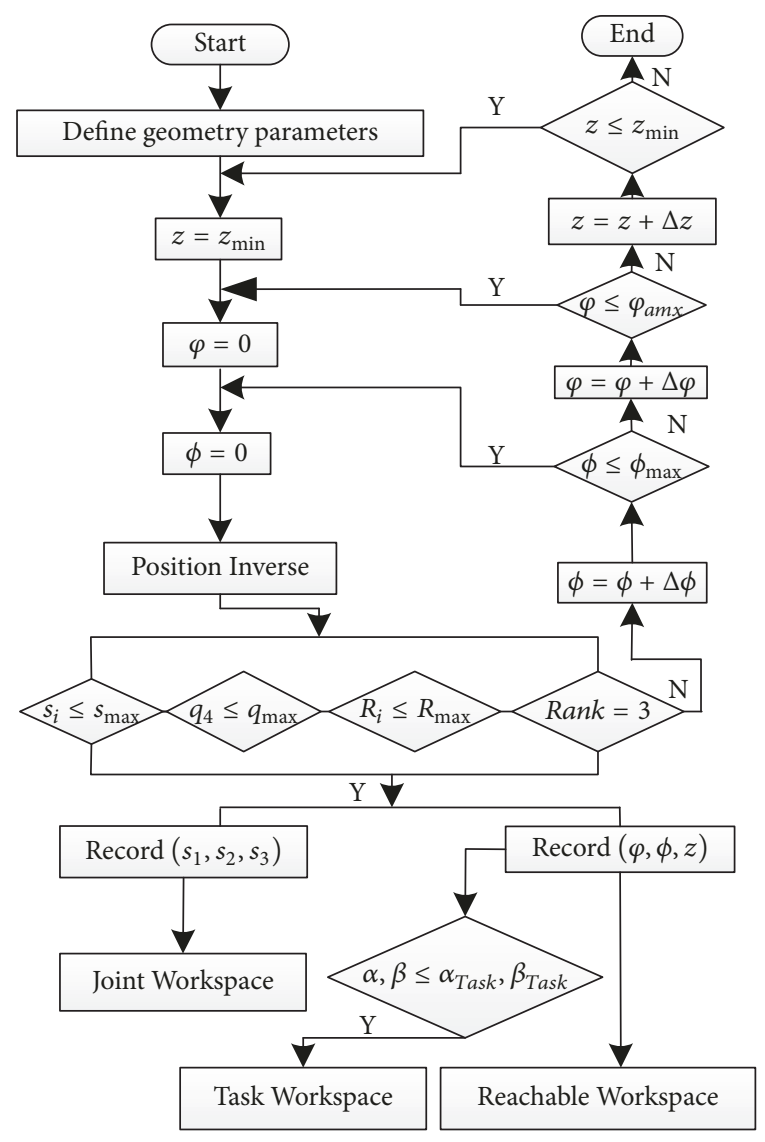

FIGURE 8: Flowchart of singularity-free workspace calculation.

fixed base radius is $b=600$, the moving platform radius is $a=300$, the length of limbs is $l_{1}=l_{2}=1045,900 \leq l_{3} \leq 1200$, and the search range of the workspace is $-4 \pi / 9 \leq \alpha \leq 4 \pi / 9$, $-\pi / 2 \leq \beta \leq \pi / 2,900 \leq z \leq 1600,-\pi / 4 \leq \alpha_{\text {Task }} \leq 0$, and $-\pi / 6 \leq \beta_{\text {Task }} \leq \pi / 6$, in terms of the relationships between Euler angle and T-T angle, the range of T-T angle is derived as $0 \leq \varphi \leq 2 \pi, 0 \leq \phi \leq 90^{\circ}$, and the figures are drawn in the cylindrical coordinate system for convenience.

6.1. Kinematic Analysis. Because the redundantly actuated and overconstrained parallel manipulator has a parasitic motion in $y$ direction, the relation mapping between $|y|$ and $(\varphi, \phi)$ is drawn in Figure 9 by $(23)$.

6.2. Performance Analysis of Orientation Workspace. To illustrate the analysis process of the orientation workspace comprising reachable workspace, task workspace, and joint workspace, the novel parallel manipulator proposed has been taken as an example. By adopting a numerical searching method aforementioned, the reachable workspace and task workspace of the redundantly actuated and overconstrained parallel manipulator are generated as shown in Figure 10; in other words, the machine tool will possess a workspace, encasing a specified task workspace. The results show that the manipulator can translate $900 \mathrm{~mm}$ to $1600 \mathrm{~mm}$ along z-axis, rotate $0^{\circ}$ to $360^{\circ}$ about $\varphi$, and rotate $0^{\circ}$ to $90^{\circ}$ about $\phi$. 


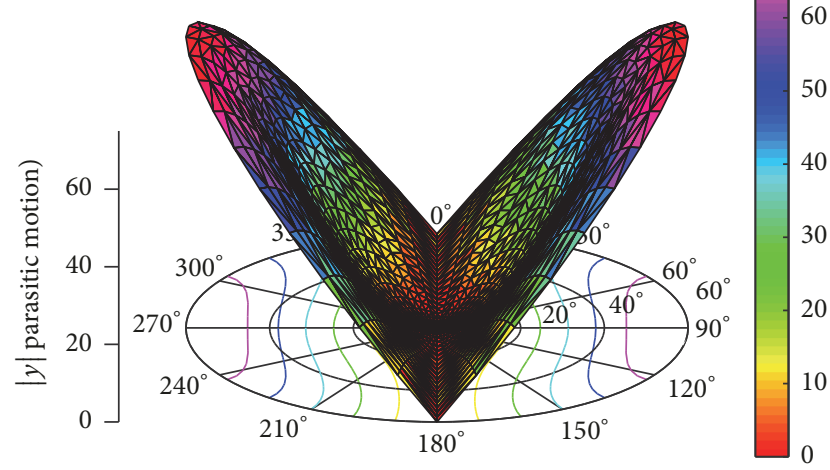

Figure 9: Parasitic motion relation between $|y|$ and $(\varphi, \phi)$ with $z=1300$.

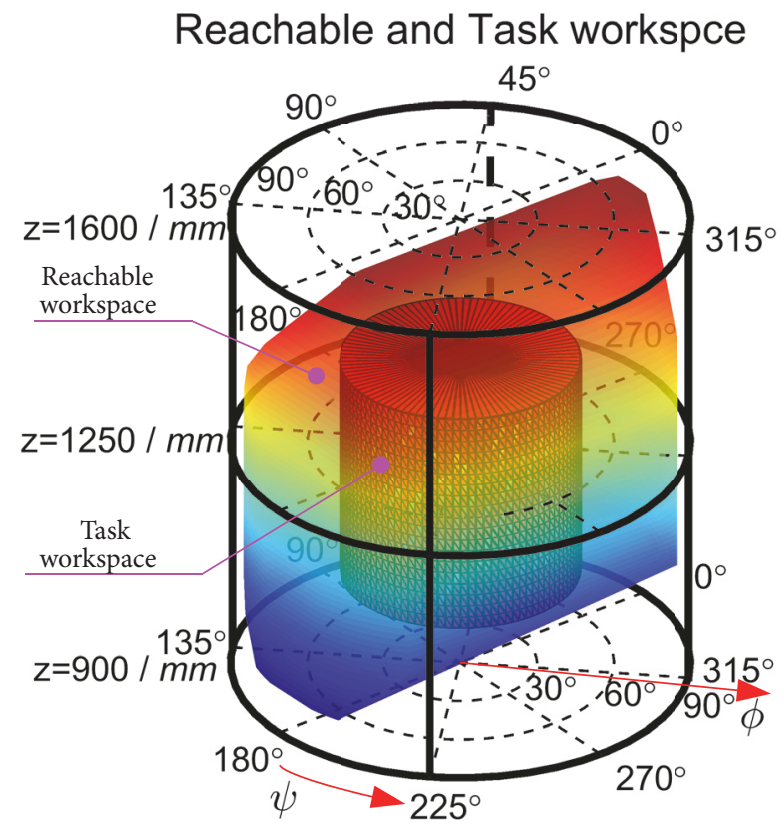

FIgURE 10: Singularity-free reachable and task workspace.

By specifying the value of the $q_{4}$ (that is link length of $l_{3}$ ), we can get a surface in three-dimensional joint workspace $\left(s_{1}, s_{2}, s_{3}\right)$ formed by the three prismatic joints. And the surface contains all the possible actuated parameters which affect the motion of the moving platform. Figure 11 shows the joint workspace when $q_{4}=1150 \mathrm{~mm}$, which gives us information that various constraints can result in a set of unreachable points and implies that the capability of motions can be efficiently utilized.

The reachable workspace of the proposed parallel manipulator and the overconstrained 2PRU-PRS parallel manipulator, with the same degree of freedom, is illustrated in Figure 12. To facilitate analysis, the same search space is used for comparison of the overconstrained 2PRU-PRS parallel manipulator and the proposed parallel manipulator. The

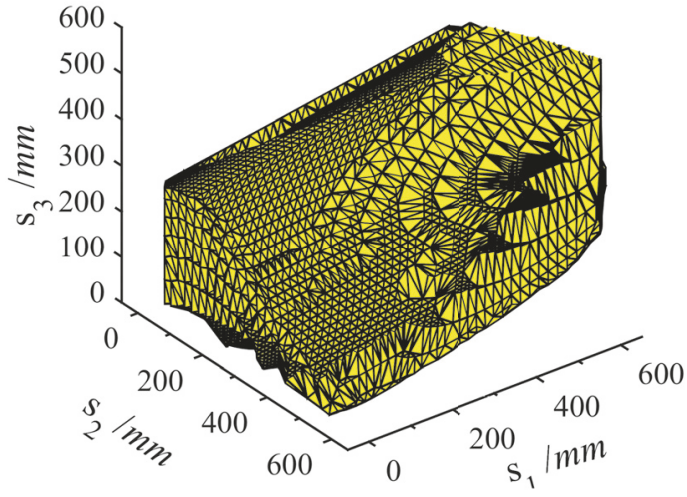

FIGURE 11: Joint workspace of the parallel manipulator.

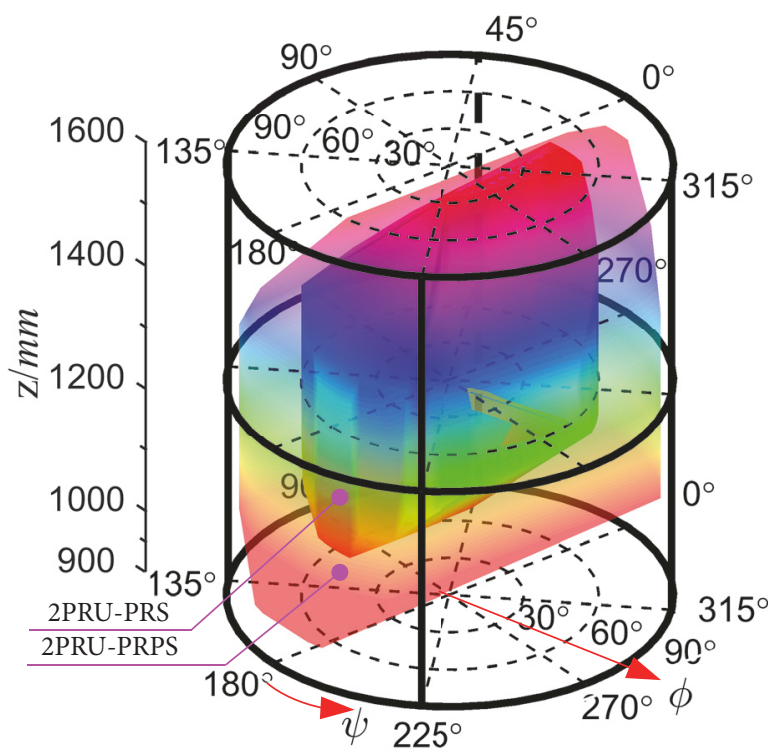

FIGURE 12: Singularity-free workspace comparison of two parallel manipulators.

result shows that the redundant actuation parallel manipulator has a larger workspace and much higher orientation rotation capability than the $2 \mathrm{PRU}-\mathrm{PRS}$ parallel manipulator. Therefore, it is feasible to select the redundantly actuated and overconstrained $2 \underline{P} R U-\underline{P} R \underline{P}$ S parallel manipulator as the main body of the hybrid machine tool and is very meaningful to the development of the five-axis hybrid machine tool.

\section{Conclusions}

In this paper, a novel serial-parallel hybrid kinematic machine tool with high stiffness, high orientation capability, and large workspace has been presented, which is a combination of 1T2R parallel manipulator and two long $\mathrm{X}-\mathrm{Y}$ tracks and can be applied to high speed machining of a large heterogeneous complex freedom surface in the aerospace field.

The mobility of proposed redundantly actuated and overconstrained 2 $\underline{P} R U$ - $\underline{P} R \underline{P} S$ parallel manipulator has been 
detailed and analyzed based on the screw theory and GrüblerKutzbach (G-K) equations, and the correctness of analysis method is verified. The inverse kinematic position and parasitic motion of the derived parallel manipulator have been sequentially analyzed, and the relationship between Z-Y-X Euler angle and Tilt-Torsion (T-T) angle is briefly expressed.

The orientation workspace, the performance evaluation index, can serve as an alternative for the description of the orientation capability. Compared with overstrained 2PRUPRS parallel manipulator, the corresponding results illustrate that the redundant actuation parallel manipulator can effectively increase the workspace and potentially improve its orientation capability, which lays a theoretical foundation for the stiffness analysis and optimal design of the parallel manipulator in the future work.

\section{Data Availability}

The data used to support the findings of this study are available from the corresponding author upon request.

\section{Conflicts of Interest}

The authors declared no potential conflicts of interest with respect to the research, authorship, and/or publication of this article.

\section{Acknowledgments}

The authors would like to acknowledge the financial support of the Fundamental Research Funds for the Central Universities under Grant no. 2018JBZ007, no. 2018YJS136, and no. 2017YJS158 and the National Natural Science Foundation of China (NSFC) under Grants 51675037 and 51505023.

\section{References}

[1] M. Terrier, A. Dugas, and J.-Y. Hascoët, "Qualification of parallel kinematics machines in high-speed milling on free form surfaces," The International Journal of Machine Tools and Manufacture, vol. 44, no. 7-8, pp. 865-877, 2004.

[2] Y. $\mathrm{Lu}$ and J. $\mathrm{Xu}$, "Computer simulation machining a 3D free surface by using a 3-RPRU parallel machine tool," The International Journal of Advanced Manufacturing Technology, vol. 33, no. 7-8, pp. 782-792, 2007.

[3] Y. D. Chen, J. Ni, and S. M. Wu, "Real-time CNC tool path generation for machining IGES surfaces," Journal of Engineering for Industry, vol. 115, no. 4, pp. 480-486, 1993.

[4] D. C. H. Yang and Z. Han, "Interference detection and optimal tool selection in 3-axis NC machining of free-form surfaces," Computer-Aided Design, vol. 31, no. 5, pp. 303-315, 1999.

[5] C.-C. Lo, "Real-time generation and control of cutter path for 5-axis CNC machining," The International Journal of Machine Tools and Manufacture, vol. 39, no. 3, pp. 471-488, 1999.

[6] Y. H. Jung, D. W. Lee, J. S. Kim, and H. S. Mok, "NC postprocessor for 5-axis milling machine of table-rotating/tilting type," Journal of Materials Processing Technology, vol. 130-131, pp. 641-646, 2002.

[7] P. Xu, C.-F. Cheung, B. Li, L.-T. Ho, and J.-F. Zhang, "Kinematics analysis of a hybrid manipulator for computer controlled ultra-precision freeform polishing," Robotics and ComputerIntegrated Manufacturing, vol. 44, pp. 44-56, 2017.

[8] Y. Lu, Y. Shi, and B. Hu, "Solving reachable workspace of some parallel manipulators by computer-aided design variation geometry," Proceedings of the Institution of Mechanical Engineers, Part C: Journal of Mechanical Engineering Science, vol. 222, no. 9, pp. 1773-1781, 2008.

[9] J. Tlusty, J. Ziegert, and S. Ridgeway, "Fundamental comparison of the use of serial and parallel kinematics for machines tools," CIRP Annals - Manufacturing Technology, vol. 48, no. 1, pp. 351356, 1999.

[10] N. Hennes and D. Staimer, "Application of PKM in aerospace manufacturing-high performance machining centers ECOSPEED, ECOSPEED-F and ECOLINER," in Proceedings of the 4th Chemnitz Parallel Kinematics Seminar, pp. 557577, Zwickau, Verlag Wissenschaftliche Scripten, Chemnitz, Germany, 2004.

[11] Y. Wang, H. Liu, T. Huang, and D. G. Chetwynd, "Stiffness modeling of the tricept robot using the overall jacobian matrix," Journal of Mechanisms and Robotics, vol. 1, no. 2, pp. 1-8, 2009.

[12] J. Zhang, Y. Q. Zhao, and Y. Jin, "Elastodynamic Modeling and Analysis for an Exechon Parallel Kinematic Machine," Journal of Manufacturing Science and Engineering, vol. 138, no. 3, 2016.

[13] B. Gherman, D. Pisla, C. Vaida, and N. Plitea, "Development of inverse dynamic model for a surgical hybrid parallel robot with equivalent lumped masses," Robotics and Computer-Integrated Manufacturing, vol. 28, no. 3, pp. 402-415, 2012.

[14] M. Weck and D. Staimer, "Parallel kinematic machine tools Current state and future potentials," CIRP Annals - Manufacturing Technology, vol. 51, no. 2, pp. 671-683, 2002.

[15] N. Hennes, "Ecospeed:an innovative machinery concept for high-performance 5-axis machining of large structural components in aircraft engineering," in Proceedings of the 3rd Chemnitz Parallel Kinematics Seminar, pp. 763-774, Zwickau, Germany, 2002.

[16] M. Wang, H. Liu, T. Huang, and D. G. Chetwynd, "Compliance analysis of a 3-SPR parallel mechanism with consideration of gravity," Mechanism and Machine Theory, vol. 84, pp. 99-112, 2015.

[17] X. Yang, J. Zhao, L. Zhang, D. Li, and R. Li, "A novel surface selfadapting parallel machine tool for blade machining," in Proceedings of the 2009 IEEE International Conference on Mechatronics and Automation, ICMA 2009, pp. 3921-3926, August 2009.

[18] Y. G. Li, H. T. Liu, X. M. Zhao, T. Huang, and D. G. Chetwynd, "Design of a 3-DOF PKM module for large structural component machining," Mechanism and Machine Theory, vol. 45, no. 6, pp. 941-954, 2010.

[19] Q. Hao, L. Guan, and L. Wang, "Intelligent acceleration/ deceleration control algorithm for drive force for a heavy duty hybrid machine tool," Qinghua Daxue Xuebao/Journal of Tsinghua University, vol. 49, no. 11, pp. 1770-1778, 2009.

[20] T. Huang, M. Li, X. M. Zhao, J. P. Mei, D. G. Chetwynd, and S. J. Hu, "Conceptual design and dimensional synthesis for a 3DOF module of the TriVariant - A novel 5-DOF reconfigurable hybrid robot," IEEE Transactions on Robotics, vol. 21, no. 3, pp. 449-456, 2005.

[21] J. Wu, J. Wang, L. Wang, T. Li, and Z. You, "Study on the stiffness of a 5-DOF hybrid machine tool with actuation redundancy," Mechanism and Machine Theory, vol. 44, no. 2, pp. 289-305, 2009.

[22] J. Fan and A. Ball, "Quadric method for cutter orientation in five-axis sculptured surface machining," The International 
Journal of Machine Tools and Manufacture, vol. 48, no. 7-8, pp. 788-801, 2008.

[23] X. Kong and M. Gosselin C, "Type synthesis of threeDOF up-equivalent parallel manipulators using a virtual-chain approach," Advances in Robot Kinematics, pp. 123-132, 2006.

[24] Q. Li and J. M. Hervé, "Type synthesis of 3-DOF RPR-equivalent parallel mechanisms," IEEE Transactions on Robotics, vol. 30, no. 6, pp. 1333-1343, 2017.

[25] Q. Li, Z. Chen, Q. Chen, C. Wu, and Z. Huang, "Structural condition for [PP]S parallel mechanism without parasitic motion," Journal of Mechanical Engineering, vol. 46, no. 15, pp. 31-35, 2010.

[26] L. Wang, H. Xu, and L. Guan, "Optimal design of a 3-PUU parallel mechanism with 2R1T DOFs," Mechanism and Machine Theory, vol. 114, pp. 190-203, 2017.

[27] X. Cui, X. Han, and W. Chen, "Continuous stiffness modeling of 3-RPS parallel kinematic machine with special composite spherical joints," Beijing Hangkong Hangtian Daxue Xuebao/Journal of Beijing University of Aeronautics and Astronautics, vol. 36, no. 11, pp. 1275-1280, 2010.

[28] B. Li, Y. Li, and X. Zhao, "Kinematics analysis of a novel overconstrained three degree-of-freedom spatial parallel manipulator," Mechanism and Machine Theory, vol. 104, pp. 222-233, 2016.

[29] F. Xie, X. Liu, and T. Li, "A Comparison Study on the Orientation Capability and Parasitic Motions of Two Novel Articulated Tool Heads with Parallel Kinematics," Advances in Mechanical Engineering, vol. 4, pp. 249103-249103, 2015.

[30] Q. Yan, B. Li, Y. Li, and X. Zhao, "Kinematics comparative study of two overconstrained parallel manipulators," Mathematical Problems in Engineering, vol. 2016, Article ID 5091405, 12 pages, 2016.

[31] A. Pashkevich, D. Chablat, and P. Wenger, "Stiffness analysis of overconstrained parallel manipulators," Mechanism and Machine Theory, vol. 44, no. 5, pp. 966-982, 2009.

[32] H. Qu, S. Guo, and Y. Zhang, "A novel relative degree-offreedom criterion for a class of parallel manipulators with kinematic redundancy and its applications," Proceedings of the Institution of Mechanical Engineers, Part C: Journal of Mechanical Engineering Science, vol. 231, no. 22, pp. 4227-4240, 2017.

[33] Z. Huang, J. Liu, and Y. Li, On mobility of mechanisms, Science Press, Beijing, China, 2011.

[34] G. Cui, H. Zhang, D. Zhang, and F. Xu, "Analysis of the kinematic accuracy reliability of a 3-DOF parallel robot manipulator," International Journal of Advanced Robotic Systems, vol. 12, pp. 1-11, 2015.

[35] X. LIU, "Attitude Description Method of [PP]S Type Parallel Robotic Mechanisms," Chinese Journal of Mechanical Engineering, vol. 44, no. 10, p. 19, 2008.

[36] Z. Z. Chi, D. Zhang, L. Xia, and Z. Gao, "Multi-objective optimization of stiffness and workspace for a parallel kinematic machine," International Journal of Mechanics and Materials in Design, vol. 9, no. 3, pp. 281-293, 2013.

[37] G. Pond and J. A. Carretero, "Quantitative dexterous workspace comparison of parallel manipulators," Mechanism and Machine Theory, vol. 42, no. 10, pp. 1388-1400, 2007.

[38] S. Herrero, C. Pinto, O. Altuzarra, and M. Diez, "Workspace study of the 2PRU-1PRS parallel manipulator," in Proceedings of the 14th International Federation for the Promotion of Mechanism and Machine Science World Congress, IFToMM 2015, October 2015.
[39] J. Fu and F. Gao, "Optimal design of a 3-leg 6-DOF parallel manipulator for a specific workspace," Chinese Journal of Mechanical Engineering, vol. 29, no. 4, pp. 659-668, 2016. 


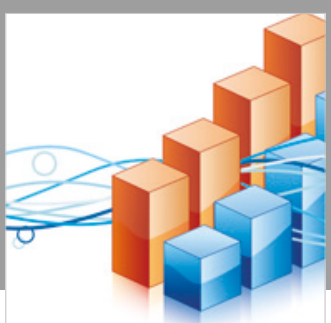

Advances in

Operations Research

\section{-n-m}
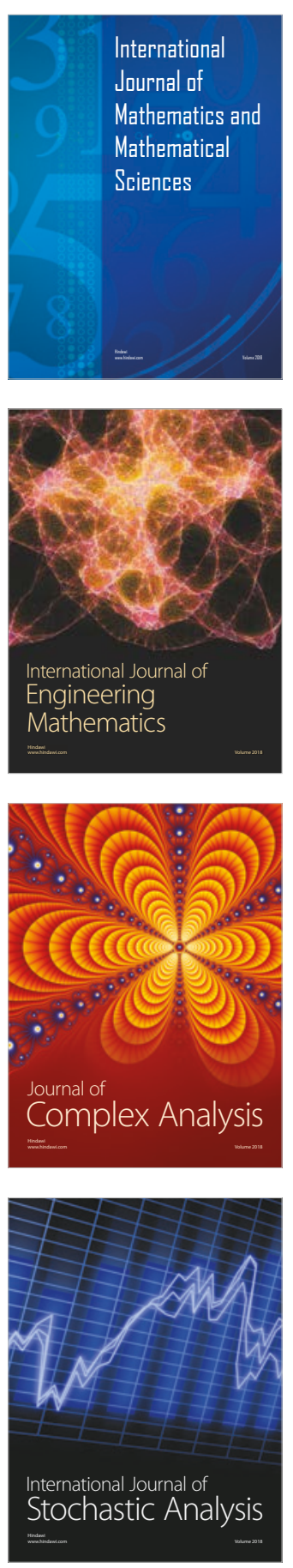
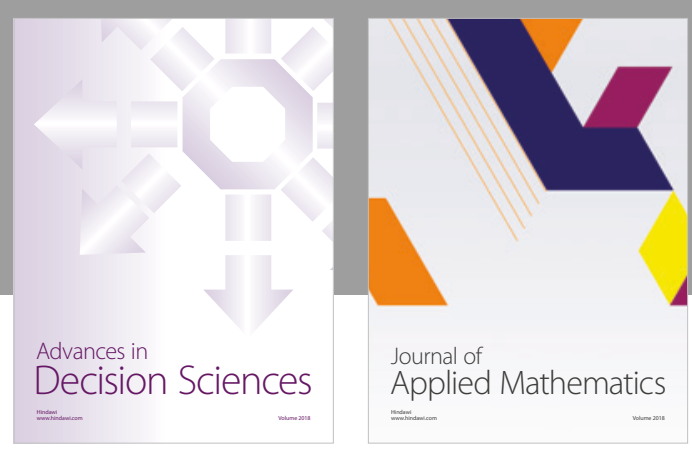

Journal of

Applied Mathematics
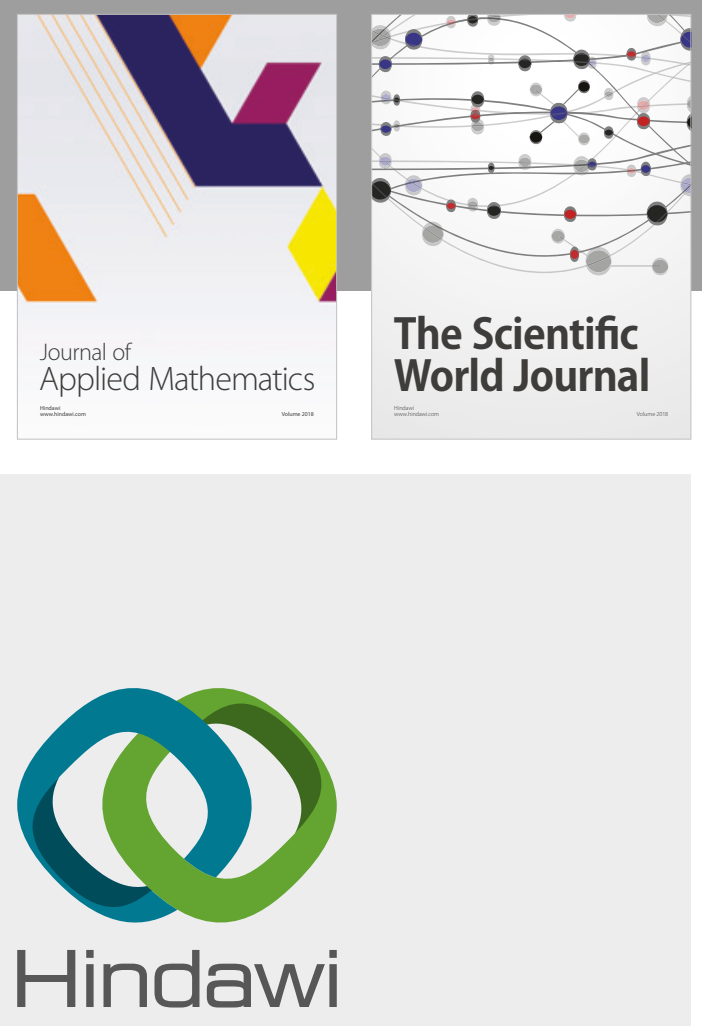

Submit your manuscripts at

www.hindawi.com

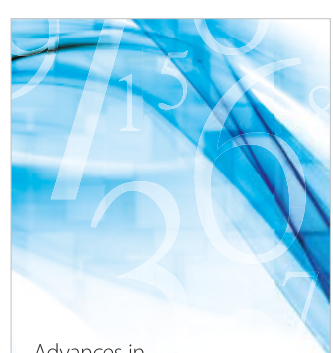

Advances in
Numerical Analysis
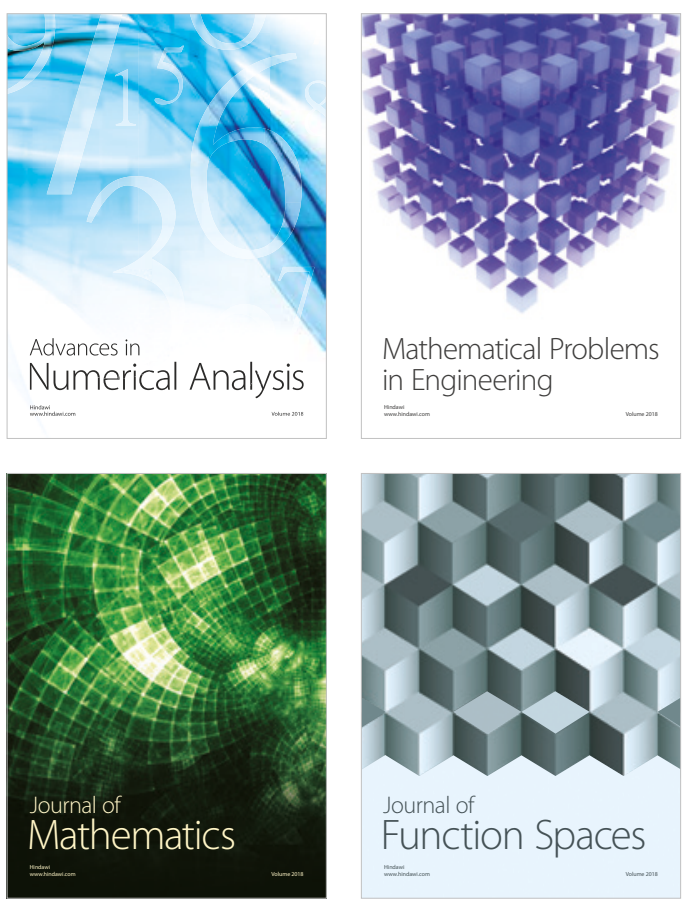

Mathematical Problems in Engineering

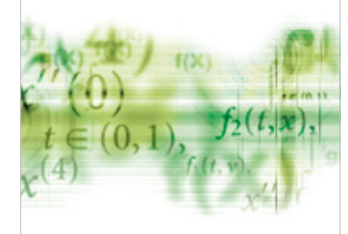

International Journal of

Differential Equations

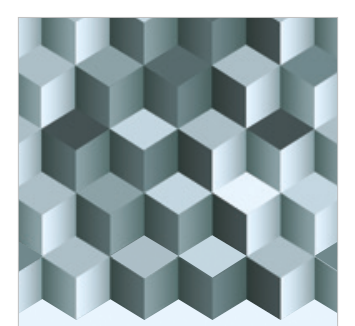

Journal of

Function Spaces

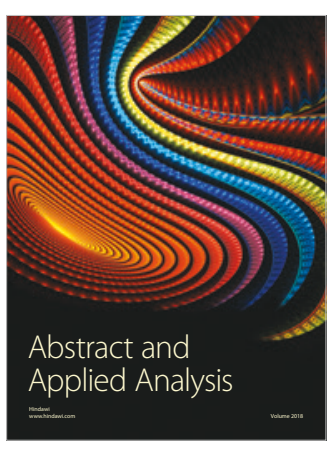

The Scientific

World Journal

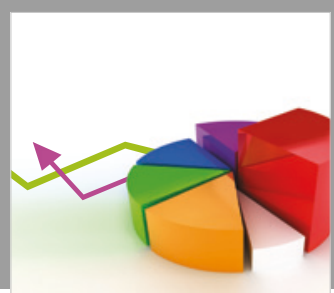

Journal of

Probability and Statistics
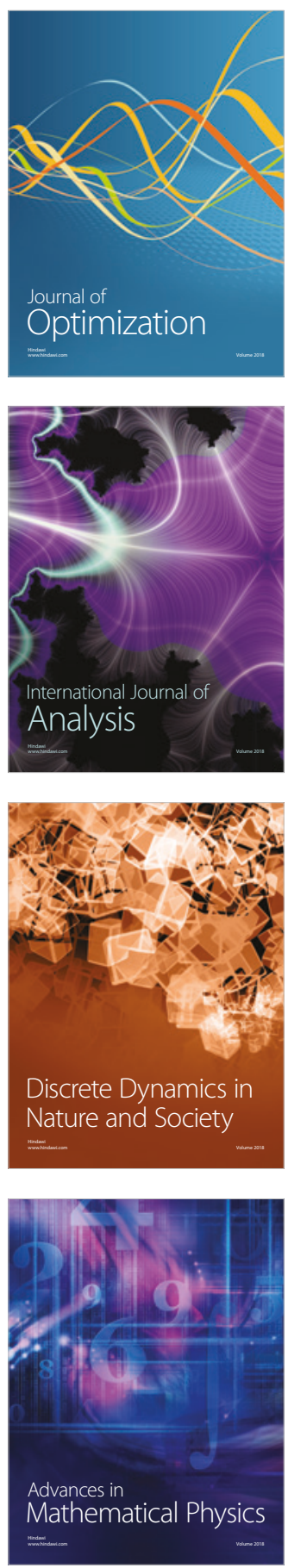